This item was submitted to Loughborough's Research Repository by the author.

Items in Figshare are protected by copyright, with all rights reserved, unless otherwise indicated.

\title{
Optimal rendezvous trajectory for unmanned aerial-ground vehicles
}

\section{PLEASE CITE THE PUBLISHED VERSION}

https://doi.org/10.1109/TAES.2017.2767958

\section{PUBLISHER}

(C) Institute of Electrical and Electronics Engineers (IEEE)

\section{VERSION}

AM (Accepted Manuscript)

\section{LICENCE}

CC BY-NC-ND 4.0

\section{REPOSITORY RECORD}

Rucco, Alessandro, Sujit P. Baliyarasimhuni, A. Pedro Aguiar, Joao Borges de Sousa, and F. Lobo Pereira. 2018. "Optimal Rendezvous Trajectory for Unmanned Aerial-ground Vehicles". figshare. https://hdl.handle.net/2134/27951. 


\title{
Optimal Rendezvous Trajectory for Unmanned Aerial-Ground Vehicles
}

\author{
Alessandro Rucco, Member, IEEE, Sujit Baliyarasimhuni, Member, IEEE, A. Pedro Aguiar, Member, IEEE, \\ João Borges de Sousa, Member, IEEE, and F. Lobo Pereira, Member, IEEE
}

\begin{abstract}
Fixed-wing unmanned aerial vehicles (UAVs) can be an essential tool for low cost aerial surveillance and mapping applications in remote regions. There is however a key limitation, which is the fact that low cost UAVs have limited fuel capacity and hence require periodic refueling to accomplish a mission. Moreover, the usual mechanism of commanding the UAV to return to a stationary base station for refueling can result in fuel wastage and inefficient mission operation time. Alternatively, one strategy could be the use of an unmanned ground vehicle (UGV) as a mobile refueling unit, where the UAV will rendezvous with the UGV for refueling. In order to accurately perform this task in the presence of wind disturbances, we need to determine an optimal trajectory in 3D taking UAV and UGV dynamics and kinematics into account. In this paper, we propose an optimal control formulation to generate a tunable UAV trajectory for rendezvous on a moving UGV that also addresses the possibility of the presence of wind disturbances. By a suitable choice of the value of an aggressiveness index that we introduce in our problem setting, we are able to control the UAV rendezvous behavior. Several numerical results are presented to illustrate the reliability and effectiveness of our approach.
\end{abstract}

Index Terms-Unmanned aerial vehicle, Unmanned ground vehicle, Rendezvous, Nonlinear optimal control, Trajectory optimization.

\section{INTRODUCTION}

$\mathbf{F}$ IXED-wing unmanned aerial vehicles (UAVs) are essential components of remote monitoring applications like surveillance, mapping, aerial photography, where the UAVs need to cover large regions. Typical UAVs used for these applications are of low cost with limited fuel capacity and hence require periodic refueling to accomplish the mission. In these scenarios, airborne docking for mid-air refueling may play an important role and has become recently a major research area, see e.g., [1], [2], [3]. However, the wake effects of the tanker on the UAV makes the analysis and design of the control scheme particularly challenging. In [4], [5], a passive towed cable system is used to retrieve the UAV, thus avoiding wake phenomena. On the other hand, a robust vision tracking method is required for the UAV to overcome some hardware

Alessandro Rucco is with the Department of Engineering, Università del Salento, Lecce, 73100, Italy, e-mail: alessandro.rucco@unisalento.it.

P.B. Sujit is with Loughborough University, Epinal Way, Loughborough LE11 3TU, UK, and IIIT Delhi, New Delhi 110020, India

A. Pedro Aguiar, João Borges de Sousa and F. Lobo Pereira are with Department of Electrical and Computer Engineering, Faculty of Engineering, University of Porto, 4200-465, Portugal, e-mail: \{pedro.aguiar, jtasso, flp\}@fe.up.pt.

This work is partially funded from Infosys Center for Artificial Intelligence, IIIT Delhi.

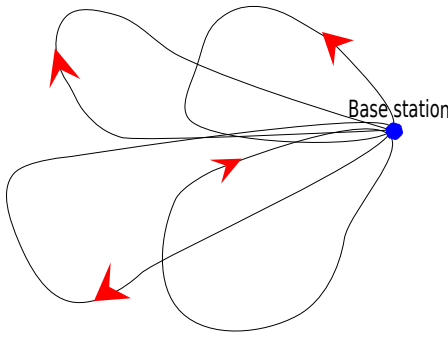

(a)

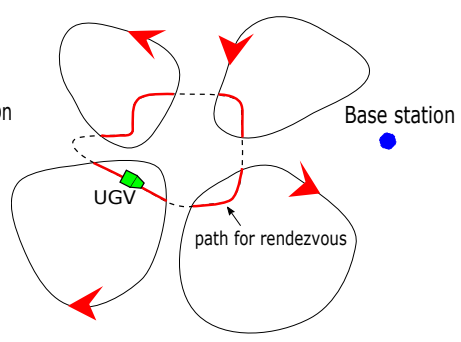

(b)
Fig. 1. (a) A field deployment where UAVs visit a base station located at a distant for refueling. (b) A UGV is deployed for refueling with a predefined UGV path and the time for rendezvous.

limitations of the vision system (mostly when the UAV gets closer to the drogue). The most simple solution is to deploy an immobile base station in a fixed location to oversee the operation and to refuel the UAVs, as shown in Figure 1a. The base station may be located at a distant which diminishes the utility of the UAVs fuel per mission. Instead of an immobile unit, an unmanned ground vehicle (UGV) can be deployed that can refuel the UAVs at different locations, and hence reducing the UAV refueling time, which increases the coverage area per refuel as shown in Figure 1b. In order to accomplish this capability, there is a need to develop techniques for UAV rendezvous with the moving UGV.

Cooperative UAV and UGV teams have been previously used for several surveillance applications. For instance, the UAV can provide useful information (e.g., data from aerial images) to the UGV for path planning and target detection [6], [7], [8], [9]. In a different application, Tokekar et al. [10] used an UAV to acquire points of nitrogen sampling in a field and the UGV used these points to create a path of one-in-aset. In this paper, we are concerned about using the UGV as a refueling mobile station and hence the UAV needs to generate a trajectory such that it can rendezvous with the moving UGV.

The UAV, UGV rendezvous can be considered either as a docking or landing problem. Aerial rendezvous between multiple aircrafts for refueling [11], [12] and formation flight [13], [14], [15] are related but the type of vehicles taken into account are not the same and the rendezvous typically is in 2D, unlike the landing, which is in 3D. Carnes et al. [16] developed an auto-takeoff and auto-landing capabilities for a low-cost UAV, which is essential for many of the envisioned applications. Nonetheless, the trajectories are not optimized, which is one of the key contributions of this paper. Kim et al. [17] developed a vision based net-landing controller for a UAV. 
The controller is based on pure-pursuit guidance law. Daly et al. [18] developed a landing controller for a quad-rotor which can hover and land on a moving vehicle. However, landing using a fixed-wing aerial vehicle instead of a quad-rotor onto a moving vehicle is much more challengeable [19]. Another relevant literature is the rendezvous/landing guidance with impact angle constraints where the impact angle is the angle about which the landing or rendezvous takes place [20], [21], [22], [23]. In those works, the trajectories are not optimized.

The contributions of this paper are as follows. First, we propose an optimization-based strategy for the generation of optimal UAV rendezvous trajectory onto a moving UGV. In order to generate realistic rendezvous trajectories, the strategy has to explicitly take into account the dynamics and kinematics of the UAV and UGV. The coupled UAV-UGV dynamics and the constraints arising from the rendezvous maneuver make the design of the strategy complex. We set up the rendezvous optimal control problem in terms of a suitable error dynamics which describe the coupled dynamics. The error dynamics make the analysis and design of the rendezvous strategy simpler, because the key for achieving successful rendezvous is that the error coordinates are zero at the rendezvous point. Second, we identify an aggressiveness index in our rendezvous optimal control problem which allows us to control the UAV rendezvous behavior. The aggressiveness index is based on the performance limitations of the UAV (i.e., the constraint limits on the state, input variables), thus allowing us to compute aggressive trajectories (several dynamic constraints are active while the UAV is approaching the UGV) or very smooth ones. The proposed optimal solution framework for the UAV-UGV rendezvous can be seen as a framework which allows one to select (in form of tuning knob) the type of UAV trajectory. Finally, through numerical computations, we show the effectiveness of our approach and discuss a set of interesting features of the rendezvous trajectories. We provide an application scenario in which we validate the proposed strategy. Given the (local) optimal trajectory, the flight path angle, the course angle, the ground speed, and the desired altitude are set as reference signals to the autopilot of a complete six-degree-of-freedom, twelve-state equations of motion, UAV model, [24]. We show that the aggressive trajectory of the 3D point mass model is "close" to the trajectory of the complex UAV model. A preliminary work on the UAV-UGV rendezvous problem was proposed by the authors in [25]. In this paper, we have extended the framework to address wind disturbances in the UAV-UGV coupled error dynamics, and have reformulated the optimal control problem by introducing an aggressiveness index term through which the complete UAV rendezvous behavior can be controlled compared with the 13 terms used in [25], and we have carried out numerical computations analyzing the effect of the aggressiveness index.

The rest of the paper is organized as follows. In Section II, we propose the optimal control formulation for the UAVUGV rendezvous. In Section III, we describe the optimal control based strategy for effectively solving the rendezvous optimal control problem. This technique is evaluated through numerical computations and illustrated in Section IV. The conclusions are given in Section V.

\section{Problem Formulation}

In this section, we address the fixed-wing UAV and UGV rendezvous problem in which the latter is moving in a plane. We first introduce the equations of motion for UAV and UGV, and outline the constraints. Next, we describe the UAV and UGV dynamics with respect to a suitable error dynamics (i.e., the velocity frame of the UGV). We then formulate the rendezvous problem with respect to the coupled UAV-UGV dynamics. In Appendix, Table I describes a list of the symbols used in the paper.

\section{A. UAV dynamic model}

We use a 3D point mass model for the aerial vehicle [24]. The six DOF equations of motion can be written as

$$
\begin{aligned}
\dot{x}_{A} & =v_{A} \cos \chi_{A} \cos \gamma_{A}, \\
\dot{y}_{A} & =v_{A} \sin \chi_{A} \cos \gamma_{A}, \\
\dot{z}_{A} & =-v_{A} \sin \gamma_{A}, \\
\dot{v}_{A} & =\frac{u_{1}-D}{m}-g \sin \gamma_{A}, \\
\dot{\gamma}_{A} & =\frac{1}{v_{A}}\left(\frac{L \cos \phi_{A}}{m}-g \cos \gamma_{A}\right), \\
\dot{\chi}_{A} & =\frac{1}{v_{A} \cos \gamma_{A}}\left(\frac{L \sin \phi_{A} \cos \left(\chi_{A}-\psi_{A}\right)}{m}\right), \\
\dot{\phi}_{A} & =u_{2},
\end{aligned}
$$

where, $L=\frac{1}{2} \rho v_{a}^{2} S C_{L}, D=\frac{1}{2} \rho v_{a}^{2} S C_{D}$, and $C_{D}=C_{D_{0}}+$ $K_{D / L} C_{L}^{2}$. The airspeed, $v_{a}$, and the ground speed, $v_{A}$, are related by

$$
\begin{aligned}
v_{A} \cos \chi_{A} \cos \gamma_{A} & =v_{a} \cos \psi_{A} \cos \gamma_{a}+w_{x}, \\
v_{A} \sin \chi_{A} \cos \gamma_{A} & =v_{a} \sin \psi_{A} \cos \gamma_{a}+w_{y}, \\
-v_{A} \sin \gamma_{A} & =-v_{a} \sin \gamma_{a}+w_{z} .
\end{aligned}
$$

where $w_{x}, w_{y}$ and $w_{z}$ are the wind components in the inertial frame. Exploiting the wind triangle, see Figure 2, the airspeed, the heading angle, and the air-mass-referenced flight path angle are given by

$$
\begin{gathered}
v_{a}=\left[v_{A}^{2}-2 v_{A}\left(w_{x} \cos \chi_{A} \cos \gamma_{A}+w_{y} \sin \chi_{A} \cos \gamma_{A}-\right.\right. \\
\left.\left.w_{z} \sin \gamma_{A}\right)+v_{w}^{2}\right]^{1 / 2}, \\
\gamma_{a}=\arcsin \left(\frac{v_{A} \sin \gamma_{A}+w_{z}}{v_{a}}\right), \\
\psi_{A}=\chi_{A}-\arcsin \left(\frac{-w_{x} \sin \chi_{A}+w_{y} \cos \chi_{A}}{v_{a} \cos \gamma_{a}}\right) .
\end{gathered}
$$

We consider three control inputs for the UAV: $u_{1}=T, u_{2}=$ $\dot{\phi}_{A}$, and $u_{3}=C_{L}$. In particular, we act on the thrust of the vehicle to affect the airspeed of the UAV. The $u_{2}$ is the roll rate by which the UAV heading angle and the flight path angle are updated. The $u_{3}$ is the lift coefficient, which we assume to operate in the linear region and hence it is considered to be approximately a linear function of the angle of attack $\alpha$ [24].

We consider that the UAVs have state and input constraints as follows. The airspeed, $v_{a}$, the load factor, $n_{l f}=\frac{L}{m g}$, and the flight path angle, $\gamma_{A}$, are bounded by $v_{\min }$ and $v_{\max }$, 


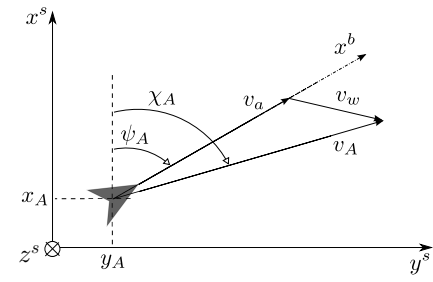

(a) Wind triangle projected onto the $x-y$ plane.

Fig. 2. The wind triangle.

$n_{l f \min }$ and $n_{l f \max }, \gamma_{\min }$ and $\gamma_{\max }$, respectively. The thrust is constrained to be positive and less than the maximum value $u_{1 \text { max }}$. Moreover, the roll angle, the roll rate, and the lift coefficient are bounded in module by $\phi_{\max }, u_{2} \max , u_{3 \max }$, respectively. More specifically, the following state and input constraints are imposed on the model:

$$
\begin{array}{r}
v_{\min } \leq v_{a} \leq v_{\max } \\
n_{l f \min } \leq n_{l f} \leq n_{l f \max } \\
\gamma_{\min } \leq \gamma_{A} \leq \gamma_{\max } \\
0 \leq u_{1} \leq u_{\max } \\
\left|\phi_{A}\right| \leq \phi_{\max } \\
\left|u_{2}\right| \leq u_{2 \max } \\
\left|u_{3}\right| \leq u_{3 \max }
\end{array}
$$

The UAV parameters, aerodynamic coefficients and the constraint parameters used in the paper are given in Appendix.

\section{B. UGV dynamic model}

We model the UGV as a 2D point mass model [26]. In this case, the equations of motion are

$$
\begin{aligned}
& \dot{x}_{G}=v_{G} \cos \chi_{G}, \\
& \dot{y}_{G}=v_{G} \sin \chi_{G}, \\
& \dot{v}_{G}=a_{l o n}, \\
& \dot{\chi}_{G}=v_{G} \sigma_{G} .
\end{aligned}
$$

We recall that the UGV can move on a pre-determined path as the one shown in Figure 1b. Therefore, we take the control input of the UGV to be the longitudinal acceleration, $u_{4}=a_{l o n}$. The lateral acceleration can be written as $a_{\text {lat }}=v_{G}^{2} \sigma_{G}$, where $\sigma_{G}$ is the (fixed, albeit time varying) path curvature [27]. Note that we describe the UGV curvature as a function of the path coordinate (or arc length coordinate) $s_{G}(t)=\int_{0}^{t} \sqrt{\dot{x}_{G}(\tau)+\dot{y}_{G}(\tau)} d \tau$. In other words, the UGV can accelerate/decelerate along the fixed path defined by the curvature.

Due to the tire-road force interaction, the vehicle acceleration is limited by the so called friction circle (more generally friction ellipse) [28]. Here, we take into account a circular acceleration constraint: the acceleration has to be less than or equal to $a_{\max }$, i.e.,

$$
a_{\text {lon }}^{2}+a_{\text {lat }}^{2} \leq a_{\max }^{2} .
$$

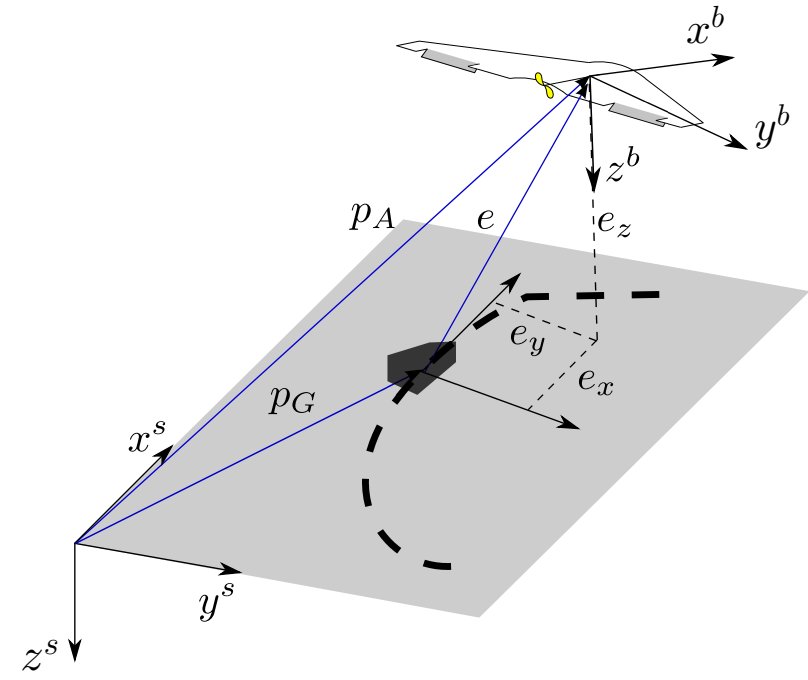

Fig. 3. Error space frames and the fixed-wing UAV body frame.

\section{Error dynamics}

From Figure 3, one can conclude that the coordinates of the aerial vehicle expressed in the inertial frame, $p_{A}=$ $\left[x_{A}, y_{A}, z_{A}\right]^{T}$, can be defined with respect to the position of the ground vehicle, $p_{G}=\left[x_{G}, y_{G}, z_{G}\right]^{T}$ as

$$
p_{A}=p_{G}+R_{z}\left(\chi_{G}\right) e,
$$

where $e=\left[e_{x}, e_{y}, e_{z}\right]^{T}$ is the error vector expressed in the body-frame of the UGV and

$$
R_{z}\left(\chi_{G}\right)=\left[\begin{array}{ccc}
\cos \chi_{G} & -\sin \chi_{G} & 0 \\
\sin \chi_{G} & \cos \chi_{G} & 0 \\
0 & 0 & 1
\end{array}\right]
$$

is the rotation matrix transforming vectors from the error frame (i.e., the velocity frame of the UGV) into the inertial frame. It is worth noting that, since the altitude of UGV is constant and equal to zero $\left(z_{G}=0\right)$, the vertical error coordinate is equal to the altitude of the UAV, i.e., $e_{z}=z_{A}$.

Next, we compute the expression of $\dot{e}=\left[\dot{e}_{x}, \dot{e}_{y}, \dot{e}_{z}\right]^{T}$. By differentiating (6) with respect to the time $t$, we get

$$
\dot{p}_{A}=\dot{p}_{G}+\left[\begin{array}{ccc}
-\sin \chi_{G} & -\cos \chi_{G} & 0 \\
\cos \chi_{G} & -\sin \chi_{G} & 0 \\
0 & 0 & 0
\end{array}\right] \dot{\chi}_{G} e+R_{z}\left(\chi_{G}\right) \dot{e}
$$

By using the kinematics of the UAV, (1), and the UGV, (4), equation (7) becomes

$$
\dot{e}=R_{z}\left(\chi_{G}\right)^{T} R_{z}\left(\chi_{A}\right) R_{y}\left(\gamma_{A}\right)\left[\begin{array}{c}
v_{A} \\
0 \\
0
\end{array}\right]-\left[\begin{array}{c}
\left(1-e_{y} \sigma_{G}\right) v_{G} \\
e_{x} \sigma_{G} v_{G} \\
0
\end{array}\right] .
$$

Equation (8) describes the kinematic position error of the UAV with respect to the UGV. Defining the course angle error as $e_{\chi}=\chi_{A}-\chi_{G}$, the speed error as $e_{v}=v_{A}-v_{G}$, the flight path error as $e_{\gamma}=\gamma_{A}$, and the roll error as $e_{\phi}=\phi_{A}$, the coupled nonlinear system (1), (4), can be written with respect to the new set of coordinates $(\mathbf{x}, \mathbf{u})=$ 


$$
\begin{aligned}
\left(e_{x}, e_{y}, e_{z}, e_{v}, e_{\gamma}, e_{\chi}, e_{\phi}, v_{G}, s_{G}, u_{1}, u_{2}, u_{3}, u_{4}\right) \text { as } \\
\dot{e}_{x}=\left(e_{v}+v_{G}\right) \cos e_{\chi} \cos e_{\gamma}-\left(1-\sigma_{G} e_{y}\right) v_{G}, \\
\dot{e}_{y}=\left(e_{v}+v_{G}\right) \sin e_{\chi} \cos e_{\gamma}-e_{x} \sigma_{G} v_{G}, \\
\dot{e}_{z}=-\left(e_{v}+v_{G}\right) \sin e_{\gamma}, \\
\dot{e}_{v}=\frac{u_{1}-D}{m}-g \sin e_{\gamma}-u_{4}, \\
\dot{e}_{\gamma}=\frac{1}{\left(e_{v}+v_{G}\right)}\left(\frac{L \cos \phi_{A}}{m}-g \cos e_{\gamma}\right), \\
\dot{e}_{\chi}=\frac{1}{\left(e_{v}+v_{G}\right) \cos e_{\gamma}}\left(\frac{L \sin \phi_{A} \cos \chi_{c}}{m}\right)-\sigma_{G} v_{G}, \\
\dot{e}_{\phi}=u_{2} \\
\dot{v}_{G}=u_{4} \\
\dot{s}_{G}=v_{G} .
\end{aligned}
$$

Given the coupled UAV-UGV dynamics (9) and the constraints (3) and (5), we introduce two additional constraints. First, the vertical error coordinate, $e_{z}$, must be non positive, to avoid a potential collision of the UAV with the ground (since the UGV altitude is zero). Second, for the physical docking at the rendezvous point, we need to define a constraint on the course angle error, $e_{\chi}$. Specifically, the following two constraints are taken into account:

$$
\begin{aligned}
e_{z} & \leq 0 \\
\left|e_{\chi}\right| & \leq\left(\frac{e_{x}}{\bar{e}_{x}}\right)^{2}+\left(\frac{e_{y}}{\bar{e}_{y}}\right)^{2}+\left(\frac{e_{z}}{\bar{e}_{z}}\right)^{2}+\bar{e}_{\chi} .
\end{aligned}
$$

It is worth noting that, due to the presence of the error coordinates $e_{x}, e_{y}, e_{z}$ in the right hand side of (10b), if the $\mathrm{UAV}$ is far away from the UGV, the course angle error is bounded by a large positive number. On the other hand, at the rendezvous point, i.e., when the kinematic error components $e_{x}, e_{y}, e_{z}$ are zero, the course angle error is just bounded by the given tolerance $\bar{e}_{\chi}>0$. This constraint formulation allows us to guide the UAV and the UGV so that a successful rendezvous is achieved guaranteeing a suitable bound on the course angle error.

\section{Optimal control problem: a trajectory tracking approach for rendezvous}

We now formulate the rendezvous problem with respect to the coupled UAV-UGV dynamics (9). Motivated by the application scenario depicted in Figure 1b, we assume that the path of the UGV and the time interval for rendezvous are given. Specifically, the UGV can move along a fixed path based on the specific scenario (e.g., a pre-determined area is assigned for docking or landing task). The time interval for rendezvous enables the UGV to create a schedule for service different vehicles operating in the same area. For this purpose, the UAV must land onto the UGV between a given time interval $\left[t_{0}, T\right]$. Moreover, the UAV is considered to be aligned with the UGV at the beginning of the rendezvous maneuver (i.e., the longitudinal and lateral position errors are zero at time $t_{0}$ ). By setting this initial condition, we are able to estimate the time to rendezvous which is an important performance feature of the UAV-UGV trajectory.
In order to accomplish a successful rendezvous, we address the problem of computing rendezvous trajectories by using a nonlinear least squares trajectory optimization technique. Specifically, we consider the following optimal control problem

$$
\begin{aligned}
\min _{\mathbf{x}(\cdot), \mathbf{u}(\cdot)} \frac{1}{2} \int_{t_{0}}^{T}\left(\left\|\mathbf{x}(\tau)-\mathbf{x}^{d}(\tau)\right\|_{Q}^{2}\right. & \left.+\left\|\mathbf{u}(\tau)-\mathbf{u}^{d}(\tau)\right\|_{R}^{2}\right) d \tau \\
& +\frac{1}{2}\left\|\mathbf{x}(T)-\mathbf{x}^{d}(T)\right\|_{P_{1}}^{2}
\end{aligned}
$$

subj. to (9), dynamics constraints

$$
\text { (3), (5), (10), state/input constraints }
$$

where $\left(\mathbf{x}^{d}(\cdot), \mathbf{u}^{d}(\cdot)\right)$ is a desired curve, $t_{0}$ and $T$ are fixed, and $Q, R$ and $P_{1}$ are positive definite weighting matrices. We address problem (11) numerically by using the projection operator based Newton method for trajectory optimization (PRONTO) with barrier function relaxation, see [29], [30] or [31] for the details. PRONTO is a direct method for solving continuous time optimal control problems. When initialized with a suitable initial trajectory, PRONTO exhibits quadratic convergence rate to a local minimizer that satisfies the second order sufficient conditions for optimality. However, a naive choice of the desired curve and the initial trajectory may lead the algorithm converge to a (local) optimal trajectory that is too far from the desired curve and, therefore, prevent the successful rendezvous between the UAV and the UGV. We mitigate this issue by proposing a novel optimization-based strategy for the design of a suitable desired curve. The desired curve is based on trimming trajectories of the UAV, i.e., the set of trajectories that can be performed using appropriate constant inputs [24]. Moreover, in order to generate a tunable UGVUAV trajectory, we propose an aggressivness index based on the maximum UAV capability. The combination of these two features (i.e., design of the desired curve and choice of the aggressivness index) allows us to effectively solve the optimal control problem (11) and generate UAV-UGV trajectories which are representative of the actual vehicles behavior. Notice that a detailed description of PRONTO goes beyond the scope of this paper, while we are interested to show the effectiveness of the rendezvous strategy for the generation of optimal UAVUGV rendezvous trajectory.

\section{Rendezvous Strategy Based on A TRAJEctory OPTIMIZATION TECHNIQUE}

In this section, we describe the optimal control based strategy for UAV-UGV rendezvous.

Specifically, we propose a rendezvous strategy based on the following two features: i) define a suitable aggressiveness index based on the maximum UAV capability; ii) choose a desired state-input curve $\left(\mathbf{x}^{d}, \mathbf{u}^{d}\right)$ based on the decoupled UAVUGV dynamics.

First, we introduce the aggressiveness index. The fixed UGV path is described by the path coordinate $s_{G} \in\left[0, s_{f}\right]$, where $s_{f}$ defines the maximum space for the execution of the rendezvous maneuver. Let $s_{r}$ be the desired space for the rendezvous maneuver, such that $0<s_{r} \leq s_{f}$. Within the 


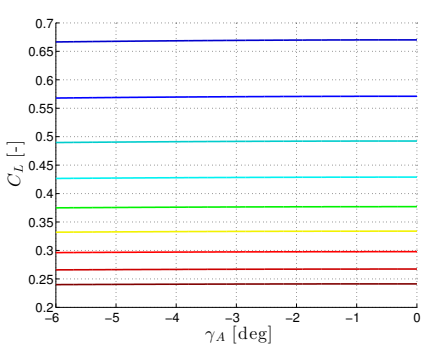

(a) Lift coefficient vs flight path angle.

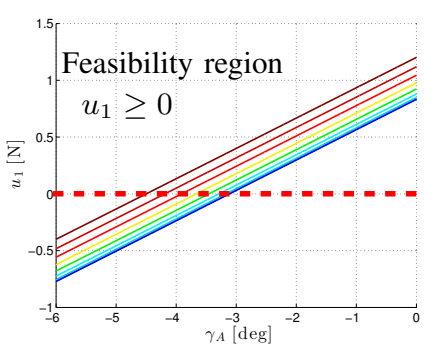

(b) Thrust vs flight path angle.

Fig. 4. Trimming trajectories of the fixed-wing UAV with $\phi_{A}=0$, $\gamma_{A}<0$ and $v_{a}=(12,13,14,15,16,17,18,19,20) \mathrm{m} / \mathrm{s}$ (blue to red): (a) lift coefficient, (b) thrust (negative thrust conditions are avoided).

rendezvous space interval $s_{G} \in\left[0, s_{r}\right]$, we set the desired constant flight path angle as

$$
\gamma_{A}^{d}=k_{\text {aggr }} \gamma_{1}+\left(1-k_{\text {aggr }}\right) \gamma_{0},
$$

where $k_{\text {aggr }} \in[0,1]$ is the aggressiveness index. The flight path angle for aggressiveness index equals to zero, i.e., $\gamma_{A}^{d}=\gamma_{0}$, is obtained by imposing the successful execution of the rendezvous maneuver at the maximum space $\left[0, s_{f}\right]$. Specifically, the rate of change of the UAV altitude can be rewritten with respect to $s_{G}$, i.e., $z_{A}^{\prime}=-\sin \gamma_{A}$, where we use the prime symbol to denote the first derivative of a variable with respect to $s_{G}$. By imposing $z_{A}\left(s_{f}\right)=0$ and integrating $z_{A}^{\prime}=-\sin \gamma_{0}$, we have $\gamma_{0}=\arcsin \left(\frac{z_{0}}{s_{f}}\right)$, where $z_{0}$ is the initial UAV altitude at which the rendezvous maneuver begins. The flight path angle for aggressiveness index equals to one, i.e., $\gamma_{1}$ in (12), is obtained by analyzing the trimming trajectories of the UAV, [24]. In that case, we are interested in forward flight with constant descent flight path angle. By setting $\dot{v}_{A}=\dot{\gamma}_{A}=0$, and $\phi_{A}=0$ in (1), we have $C_{L}=\frac{2 m g \cos \gamma_{A}}{\rho S v_{a}^{2}}$, and $u_{1}=m g \sin \gamma_{A}+\frac{1}{2} \rho S v_{a}^{2}\left(C_{D_{0}}+K_{D / L} C_{L}^{2}\right)$, see Figure 4 . As highlighted in Figure $4 \mathrm{~b}$, the thrust decreases linearly with respect to $\gamma_{A}$ and becomes negative (and, therefore, unfeasible) for $\gamma_{A}<-\frac{\rho S v_{a}^{2}}{2 m g}\left(C_{D_{0}}+K_{D / L} C_{L}^{2}\right)$ (for small values of $\gamma_{A}$ ). In order to ensure the feasibility of the desired curve, we set

$$
\gamma_{1}=-\frac{\rho S v_{\max }^{2}}{2 m g}\left(C_{D_{0}}+K_{D / L}\left(\frac{2 m g}{\rho S v_{\max }^{2}}\right)^{2}\right) .
$$

In Figure 5a we show that more close the aggressiveness index is to one, more close to the boundary constraint will be the thrust, see Figure 5a.

Second, we choose a desired state-input curve based on the decoupled UAV-UGV dynamics. Exploiting the desired flight path angle based on the aggressiveness index (12) and taking into account that the altitude of UGV is constant and equal to zero, the desired vertical error coordinate is given by

$e_{z}^{d}\left(s_{G}\right)=z_{0}-s_{G} \sin \left(k_{a g g r} \gamma_{1}+\left(1-k_{a g g r}\right) \gamma_{0}\right), s_{G} \in\left[0, s_{r}\right]$.

For UAV-UGV rendezvous, we have $e_{z}^{d}\left(s_{r}\right)=0$ and, therefore,

$$
s_{r}=\frac{z_{0}}{\sin \left(k_{\text {aggr }} \gamma_{1}+\left(1-k_{\text {aggr }}\right) \gamma_{0}\right)} .
$$

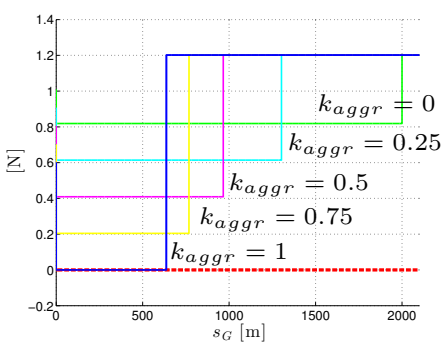

(a) $u_{1}\left(k_{\text {aggr }}\right)$.

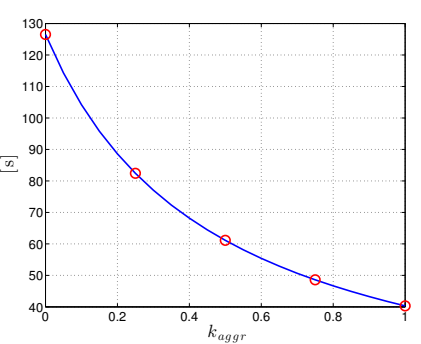

(b) $T_{r}^{d}\left(k_{\text {aggr }}\right)$.
Fig. 5. (a) Thrust and (b) rendezvous time based on the aggressiveness index $k_{\text {aggr }}=\{0,0.25,0.5,0.75,1\}$.

In order to achieve smooth "docking", the UAV has to decelerate from the initial speed, $v_{0}$, to the final speed, $v_{f}$, with $v_{\min } \leq v_{f}<v_{0}$. To this end, we set the desired speed profile as follows

$$
v^{d}\left(s_{G}\right)=v_{0}+\frac{v_{f}-v_{0}}{s_{r}} s_{G}, s_{G} \in\left[0, s_{r}\right] .
$$

The speed profile $v^{d}$ is used to time parametrize the path and generate the desired curve for the optimal control problem (11). In particular, given the space-dependent desired vertical error (13) and speed profile (14), the corresponding time-dependent desired vertical error and speed profile can be calculated by integrating $d t=d s_{G} / v^{d}$, i.e.,

$$
t\left(s_{G}\right)=\int_{0}^{s_{G}} \frac{d s_{G}}{v^{d}} .
$$

Now, it is straightforward to compute the desired rendezvous time as a function of the aggressiveness index:

$$
T_{r}^{d}=t\left(s_{r}\right)=\frac{s_{r}}{\left(v_{f}-v_{0}\right)} \ln \frac{v_{f}}{v_{0}} .
$$

As expected, increasing the aggressiveness index, the desired rendezvous time decreases, see Figure 5b. It is worth noting that, since the desired speed is strictly greater than zero (note that $v_{f}>0$ and $v_{0}>0$ ), the mapping $s_{G} \mapsto t\left(s_{G}\right)$ is strictly increasing, so that $t\left(s_{G}\right)$ is well defined.

Given the desired vertical error, UGV speed profile, and rendezvous time, next we choose the remaining state and input components of the desired curve. For successful rendezvous, the desired longitudinal and lateral error coordinates, $\left(e_{x}^{d}, e_{y}^{d}\right)$, course angle error, $e_{\chi}^{d}$, speed error, $e_{v}^{d}$, roll error, $e_{\phi}^{d}$, and flight path error, $e_{\gamma}^{d}$, are set to zero. The desired thrust and lift coefficient are chosen by exploiting UAV trim conditions [24]. In particular, assuming the UAV is in forward flight and constant-altitude flight (i.e., $\gamma_{A}=0, \phi_{A}=0$ ) with the desired UGV speed profile $v_{G}^{d}$, and under trim conditions (i.e., $\dot{v}_{A}=\dot{\gamma}_{A}=0$ in (1)), we have

$$
u_{3}^{d}=\frac{2 m g}{\rho S v_{a}^{d 2}}, u_{1}^{d}=\frac{1}{2} \rho v_{a}^{d 2} S\left(C_{D_{0}}+K_{D / L} u_{3}^{d 2}\right),
$$

where the desired airspeed, $v_{a}^{d}$, is obtained from the desired speed profile and the wind triangle relation (2). In order to set the desired thrust and lift coefficient, the wind speed and direction are assumed constant and known to the optimization solver (the wind can be estimated from sensors available in 
an autopilot module [32]). The desired UAV roll rate, $u_{2}^{d}$, and the desired UGV acceleration, $u_{4}^{d}$, are set to zero.

It is worth noting that, through the definition of the desired vertical error coordinate (13), the rendezvous problem (11) is parametrized by the aggressiveness index. The main motivation to use the aggressiveness index is twofold: predict the time-to-rendezvous (i.e., equation (16)) and provide a tool in form of tuning knob (we recall that $k_{\text {aggr }}=[0,1]$ ) which allows one to specify the aggressiveness of the UAV trajectory.

Now, with the desired curve in hand, we design the trajectory to initialize PRONTO as follows. The UAV is in forward flight, constant-altitude flight equal to $z_{0}$, and constant speed profile equal to the initial speed $v_{0}$. The UGV is traveling along the pre-determined path with constant speed equal to $v_{0}$. Given the initial UAV and UGV trajectories, the initial trajectory for the coupled UAV-UGV dynamics (i.e., it satisfies (9)) is obtained by using (6).

We highlight that the desired curve is not a trajectory (it does not satisfy the coupled UAV-UGV dynamics) whereas the initial trajectory is a non-aggressive maneuver, which is easy to compute. This is an important point of the strategy. The desired curve is in fact a guess and we leave PRONTO to take care of the dynamics and state-input constraints and thus compute a trajectory (i.e., satisfying the UAV-UGV dynamics).

\section{NUMERICAL COMPUTATIONS}

We illustrate the proposed UAV-UGV rendezvous strategy using numerical computations. We start with a relatively simple benchmark scenario: the UAV is landing onto the UGV which is moving along a straight line path. Then, motivated by the scenario in Figure $1 \mathrm{~b}$, we take into account a $90^{\circ}$ turn for the UGV path: the strong coupling between longitudinal and lateral dynamics of both UAV and UGV makes the computations particularly challenging and allows us to strengthen the results. For both scenarios, the rendezvous maneuver starts at $t=50 \mathrm{sec}$. The initial ground speed is $18 \mathrm{~m} / \mathrm{s}$ and the final rendezvous speed is set to $1.15 v_{\text {min }}$. We assume planar wind field with wind components $\left(w_{x}, w_{y}, w_{z}\right)=(-4.33,2.5,0)$. It is worth noting that, differently from the approach proposed in [25], we do not tune the 13 terms in the weighing matrices (they are the same for all the computations). In order to control the aggressivness of the (local) optimal trajectory, we modify only one parameter, i.e., the aggressiveness index $k_{\text {aggr }}$.

\section{A. Rendezvous on a straight line path}

The initial position of the UAV is $\left(x_{A}, y_{A}, z_{A}\right)=$ $(0,0,-50)$, the orientation is $\chi_{A}=\pi / 4$, flight angle and roll angle are $\gamma_{A}=0, \phi_{A}=0$, respectively. The initial position and orientation of the UGV are $\left(x_{G}, y_{G}, z_{G}\right)=(0,0,0)$, and $\chi_{G}=\pi / 4$, respectively. The maximum space for the execution of the rendezvous maneuver is $s_{f}=2000 \mathrm{~m}$. We run PRONTO based on the rendezvous trajectory generation strategy for aggressiveness index equals to $k_{a g g r}=0$. The local optimal trajectory is shown in Figures 6 and 7.

In Figure 6, we show the (local) optimal 3D path traversed by the UAV to rendezvous with the UGV. The (local) optimal UAV path is soft: the UAV height is reduced gradually.

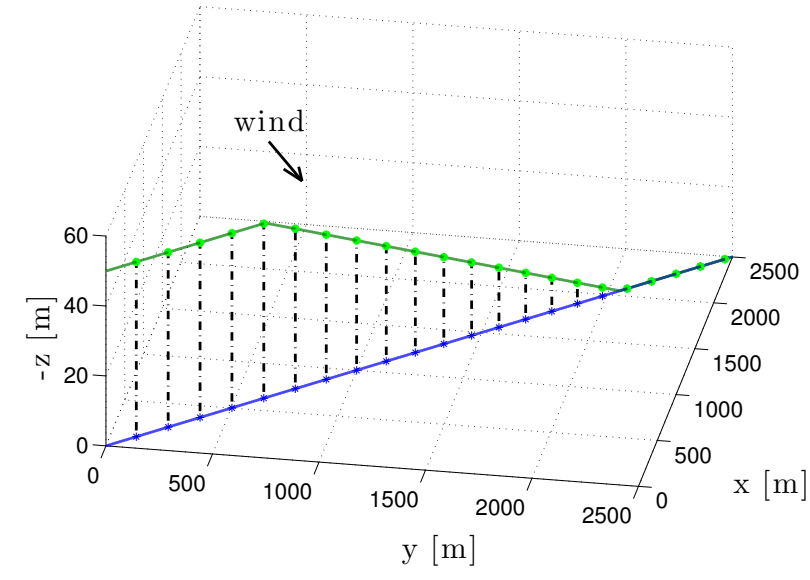

Fig. 6. Rendezvous on a straight line path for $k_{a g g r}=0$. The blue and green lines represent the UGV and the UAV paths, respectively.

This soft feature is also evident from the trajectory shown in Figure 7. Indeed, the vertical error coordinate and the flight path angle vary smoothly, Figures $7 \mathrm{a}$ and $7 \mathrm{c}$, and the constraints on thrust, flight path angle, coefficient lift, and normal load are never active, Figures 7d, 7c, 7e, 7f. We can also observe that the (local) optimal thrust is different from the desired one, see Figure 7d. Such a difference is due to the fact that the desired curve is based on trim conditions (i.e., speed transition in forward flight and constant altitude, see (17)) and, thus, does not take into account the change in the flight altitude as well as important dynamic features. Finally, we highlight that the rendezvous time is $126.7 \mathrm{sec}$ (note that $e_{z}=-0.1 \mathrm{~m}$ for $t=176.7 \mathrm{sec}$, see Figure 9a) and the desired rendezvous time is $T_{r}^{d}=126.5 \mathrm{sec}$, see (16).

We run the rendezvous trajectory generation strategy for aggressiveness index equals to $k_{\text {aggr }}=\{0.25,0.5,0.75,1\}$ and we compare the (local) optimal trajectories in Figure 8 (for the sake of completeness, we include the rendezvous trajectory obtained with $k_{\text {aggr }}=0$ ). For $k_{\text {aggr }}=1$ the (local) optimal UAV path (blue line in Figure 8a) is aggressive. By aggressive, we mean that the several constraints are active during the rendezvous maneuver. Indeed, the thrust is zero for almost all the rendezvous maneuver, Figure $8 \mathrm{~d}$, and the constraint on the normal load is active at the beginning of the maneuver, Figure 8f. Moreover, we observe a (relative) high speed error during the maneuver, Figure $8 \mathrm{~b}$. Such a difference in the ground speed between the UAV and the UGV is due to the wind (which affects only the UAV) and the fact that the airspeed is constrained.

Next, we highlight two interesting features of the (local) optimal trajectory for $k_{a g g r}=1$. First, at the beginning of the rendezvous maneuver, the UAV decreases the thrust and, at the same time, increases the lift coefficient (see the kink at about $t=50 \mathrm{sec}$ in Figure 8e, blue line). In this way, the airspeed decreases at about $t=50 \mathrm{sec}$ and, immediately after, increases thus reaching its maximum value. Such aggressive maneuver allows the UAV to take a steep dive towards the UGV as shown 


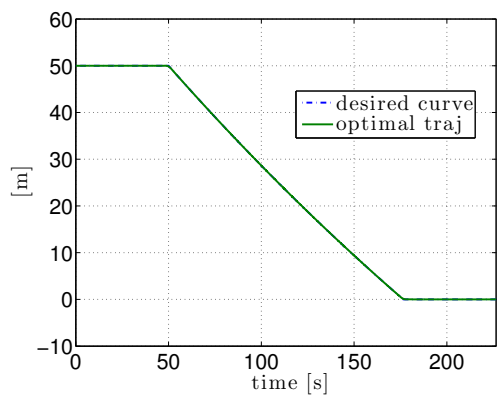

(a) $-e_{z}$.

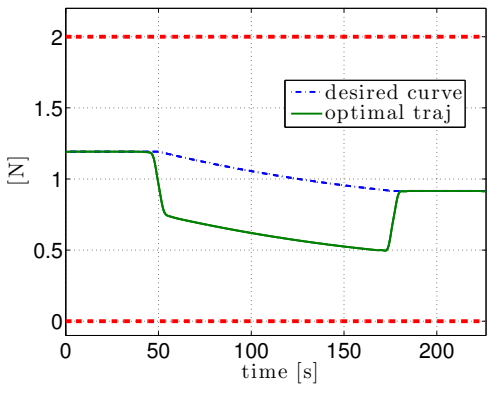

(d) $u_{1}$.

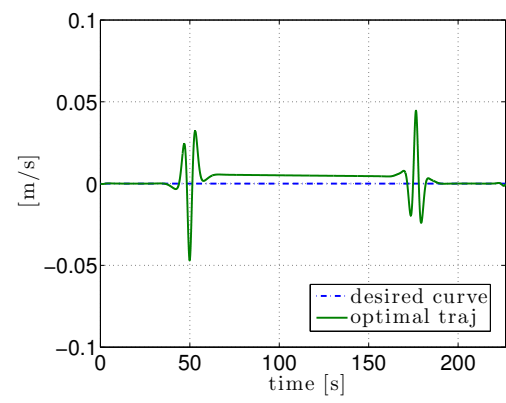

(b) $e_{v}$.

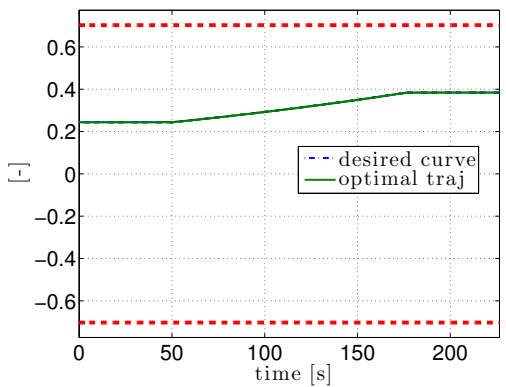

(e) $u_{3}$.

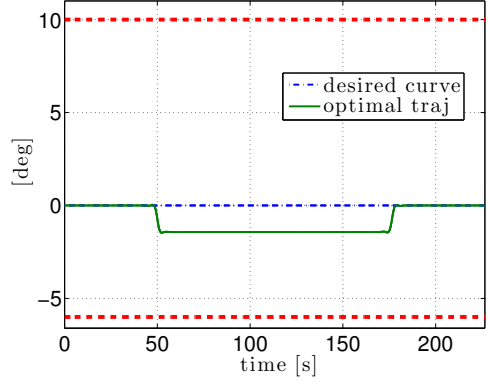

(c) $e_{\gamma}$.

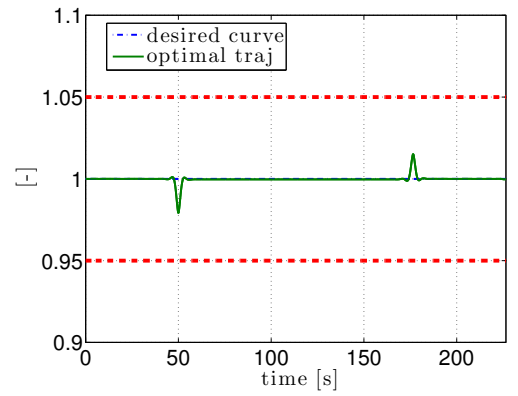

(f) $n_{l f}$.

Fig. 7. Rendezvous on a straight line path for $k_{a g g r}=0$ (a) vertical error coordinate, (b) error speed, (c) error flight path angle, (d) thrust, (e) coefficient lift, and (f) load factor. Constraints are in dashed line.

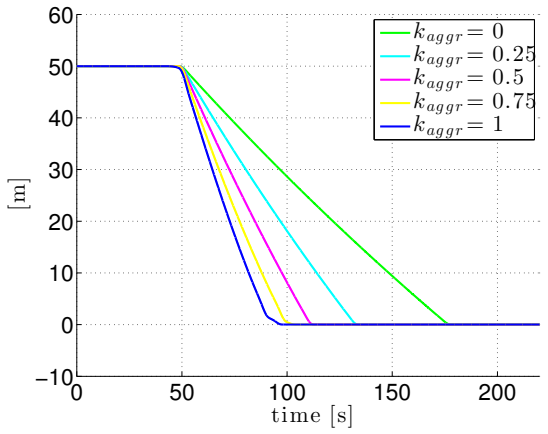

(a) $-e_{z}$.

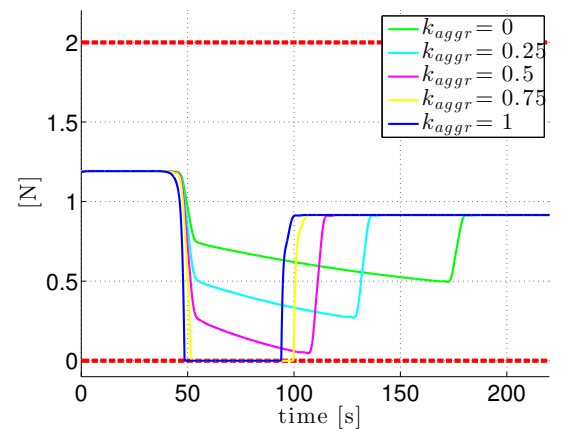

(d) $u_{1}$.

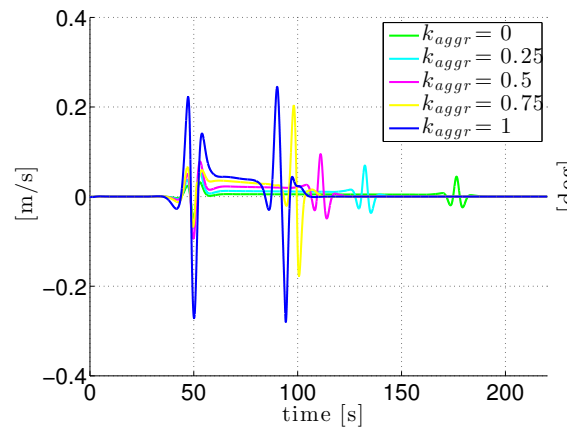

(b) $e_{v}$.

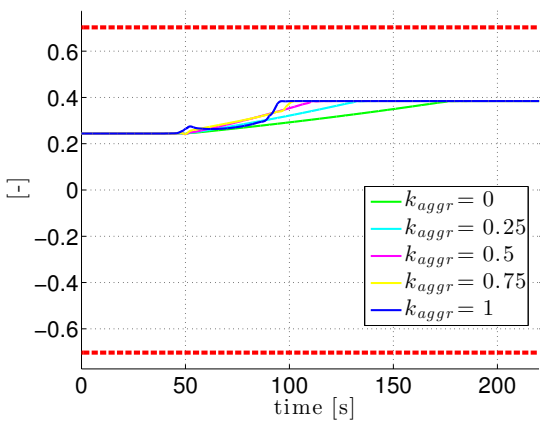

(e) $u_{3}$.

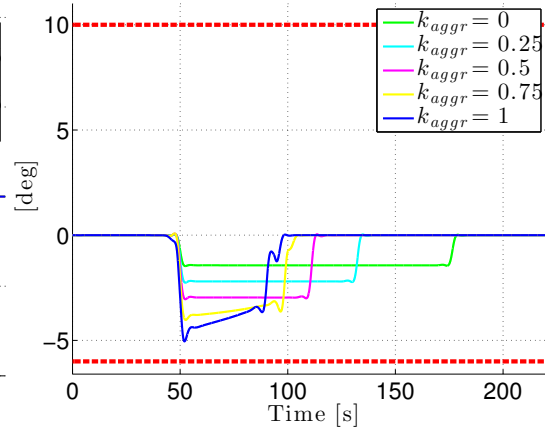

(c) $e_{\gamma}$.

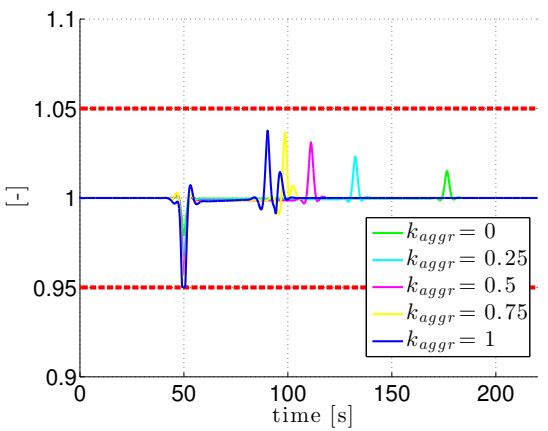

(f) $n_{l f}$.

Fig. 8. Rendezvous on a straight line path (a) vertical error coordinate, (b) error speed, (c) error flight path angle, (d) thrust, (e) coefficient lift, and (f) load factor for $k_{a g g r}=\{0,0.25,0.5,0.75,1\}$. Constraints are in dashed line. 


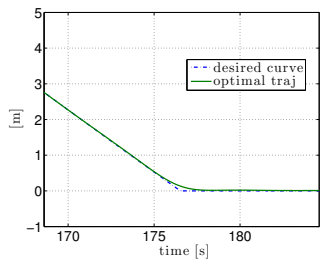

(a) $k_{\text {aggr }}=0$.

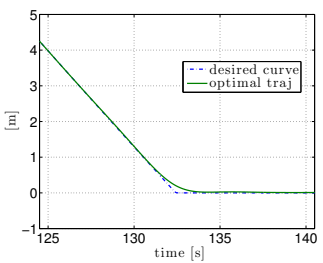

(b) $k_{\text {aggr }}=0.25$.

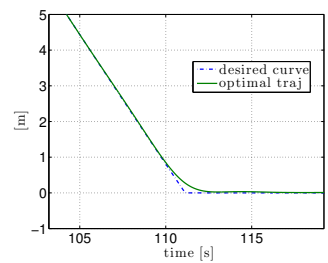

(c) $k_{\text {aggr }}=0.5$.

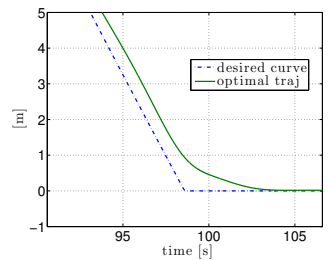

(d) $k_{\text {aggr }}=0.75$.

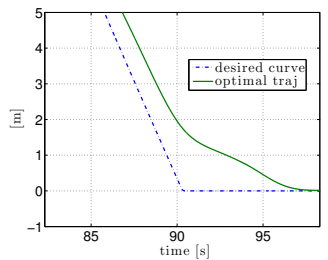

(e) $k_{\text {aggr }}=1$.

Fig. 9. The vertical error coordinate when the UAV is approaching the UGV for different $k_{\text {aggar }}$.

in Figure 8a. Second, once the UAV ground-speed reaches the desired value of $13.8 \mathrm{~m} / \mathrm{s}$ (i.e., $1.15 v_{\text {min }}$ ), the UAV needs to maintain this speed and hence it requires thrust which is increased from zero to the desired value as shown in the Figures 8d. Similar behavior is observed in the lift coefficient, Figure 8e. Such a maneuver allows the UAV to reach the UGV without overshooting, thus ensuring the feasibility of the trajectory (i.e., it satisfies the constraints (10)), see Figure 8a and the zoom in Figure 9e at about 95sec. In Figure 10 we show the trajectory of the UAV in the $x-z$ space. Starting from the constant descent flight (steep glide) to the rendezvous conditions, $e_{z}^{d}=0$, the UAV approaches the UGV with i) a very smooth altitude profile and ii) with negligible vertical velocity. From $x \in[1110,1180]$, the trajectory resembles the flare maneuver, [33].

Finally, the sequence of rendezvous time is $82.9 \mathrm{sec}, 61.8 \mathrm{sec}$, $52.4 \mathrm{sec}, 46.52 \mathrm{sec}$ for $k_{\text {aggr }}=0.25,0.5,0.75,1$, respectively. Comparing the rendezvous time with the desired one predicted by (15), we observe a good matching expect for the case $k_{\text {aggr }}=1$. In fact, due to the transient behavior at the end of the aggressive maneuver, the optimal rendezvous time is $46.52 \mathrm{sec}$ (note that $e_{z}=-0.1$ for $t=96.52 \mathrm{sec}$, see Figure 9e), yet the desired rendezvous time is $40.31 \mathrm{sec}$.

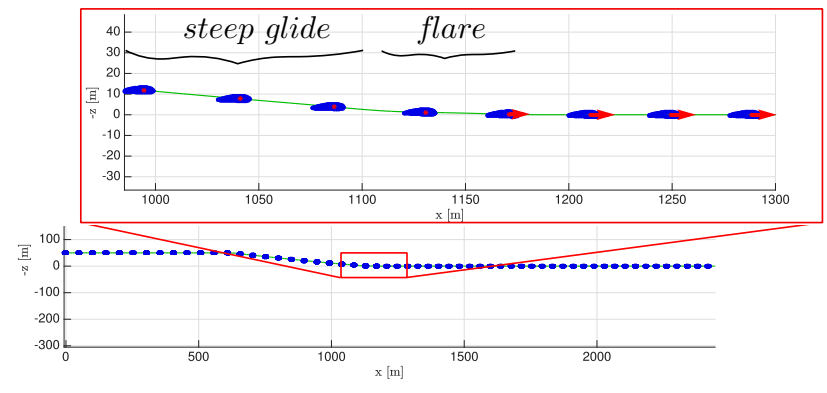

Fig. 10. Rendezvous on a straight line path for $k_{a g g r}=1$ : the $x-z$ trajectory (green line) for the fixed-wing UAV (in blue). The red lines indicate the thrust, scaled according to the thrust value.

\section{B. Rendezvous with coupled longitudinal and lateral motion}

In this scenario, the UGV is moving along a circuit as the one mentioned in the Introduction. In particular, we take into account a section of the circuit shown in Figure $1 \mathrm{~b}$ which is composed by $90^{\circ}$ turn with a radius of $35 \mathrm{~m}$ and straights of $1200 \mathrm{~m}$ before and after the turn, see Figure 11. The initial position of the UAV is $\left(x_{A}, y_{A}, z_{A}\right)=(0,0,-50)$, the orientation is $\chi_{A}=0$, and flight angle and roll angle are $\gamma_{A}=0$, $\phi_{A}=0$, respectively. The initial position and orientation of the $\mathrm{UGV}$ are $\left(x_{G}, y_{G}, z_{G}\right)=(0,0,0)$ and $\chi_{G}=0$, respectively. We run the rendezvous trajectory generation strategy for aggressiveness index equals to $k_{\text {aggr }}=\{0,0.25,0.5,0.75,1\}$ and we compare the (local) optimal trajectories in Figures 11 and 12 .

As in the previous computations, we are able to control the aggressiveness of the UAV trajectory. Thus, for $k_{\text {aggr }}=1$ (blue line in Figures 11 and 12) several constraints are active. For $k_{\text {aggr }}=0$, the UAV height is reduced gradually thus highlighting the soft feature of the local optimal trajectory (green line in Figures 11 and 12).

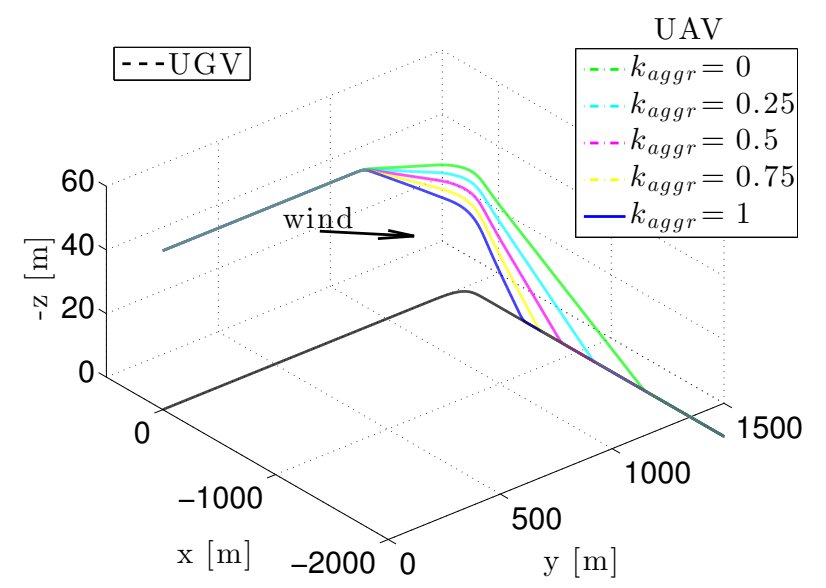

Fig. 11. Three dimension path of the UAV for a complex scenario for different $k_{\text {aggr }}=\{0,0.25,0.5,0.75,1\}$.

It is worth highlighting the effect of the right turn on the rendezvous maneuver. In order to minimize the lateral error coordinate, the UAV turns by rolling, see Figure 13b. However, the UAV is not able to track exactly the UGV. The lateral error coordinate is no zero and the constraint on the roll angle is never active, see Figures 13a and 13b. This is due to the fact that the constraint on the load factor becomes active (see Figure 12f) before the roll angle reaches its maximum value. Indeed, in constant descent flight conditions (i.e., $\dot{\gamma}_{A}=0$ and $\gamma_{A}<0$ ), the lift must be equal to $m g \frac{\cos \gamma_{A}}{\cos \phi_{A}}$ and the load 


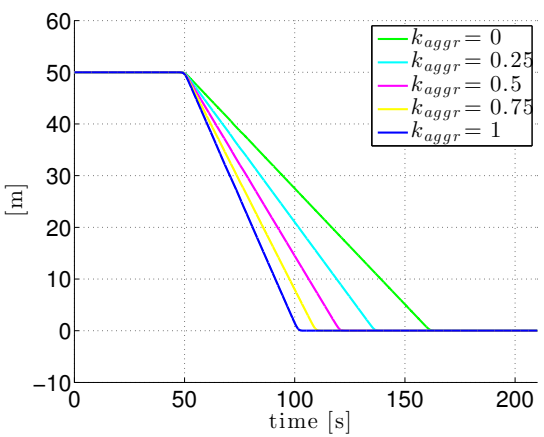

(a) $e_{z}$.

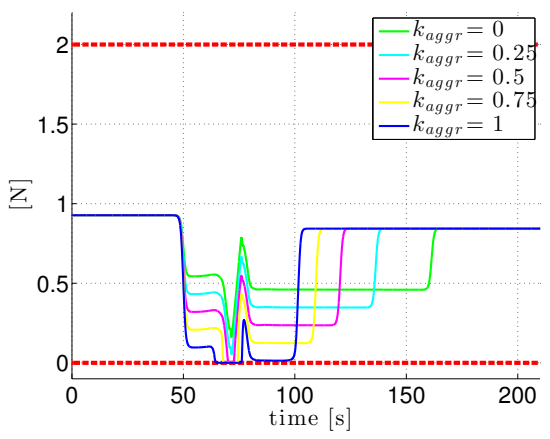

(d) $u_{1}$.

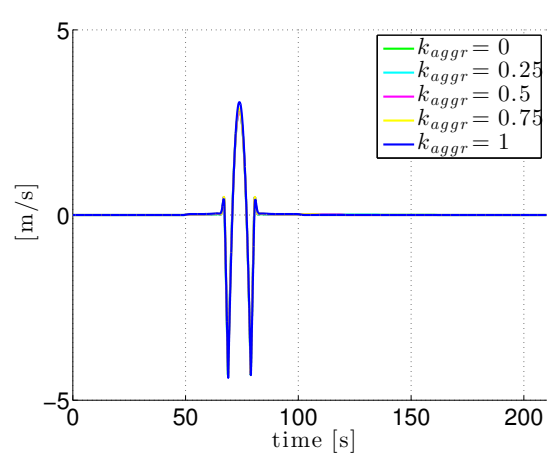

(b) $e_{v}$.

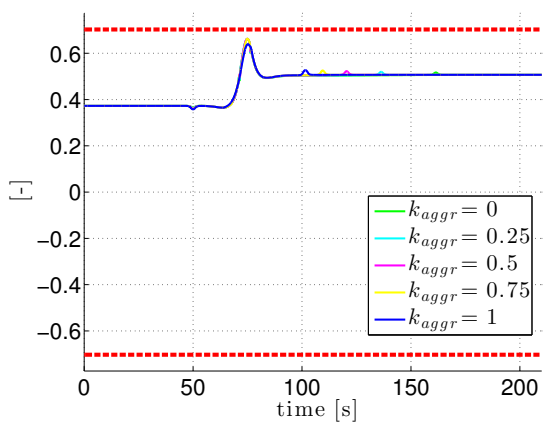

(e) $u_{3}$.

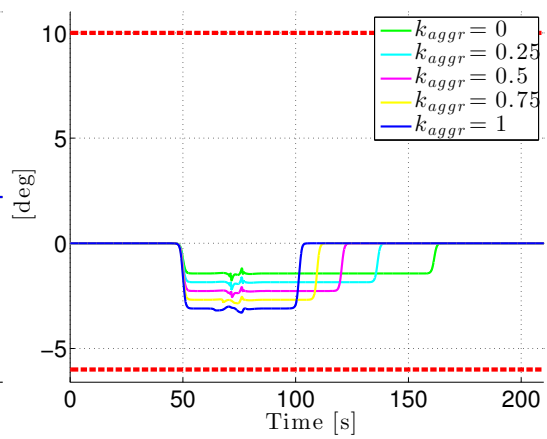

(c) $e_{\gamma}$.

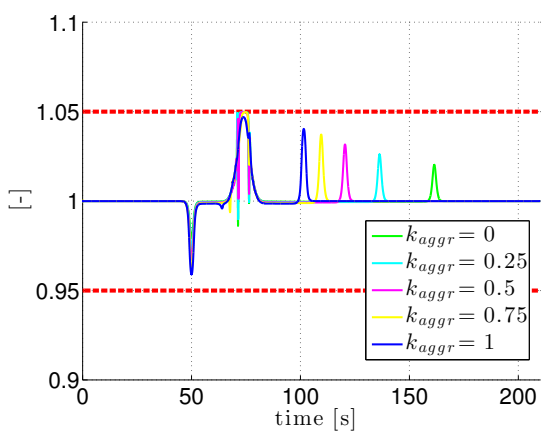

(f) $n_{l f}$.

Fig. 12. Rendezvous with coupled longitudinal and lateral motion (a) vertical error coordinate, (b) error speed, (c) error flight path angle, (d) thrust, (e) coefficient lift, and (f) load factor for $k_{a g g r}=\{0,0.25,0.5,0.75,1\}$. Constraints are in dashed line.

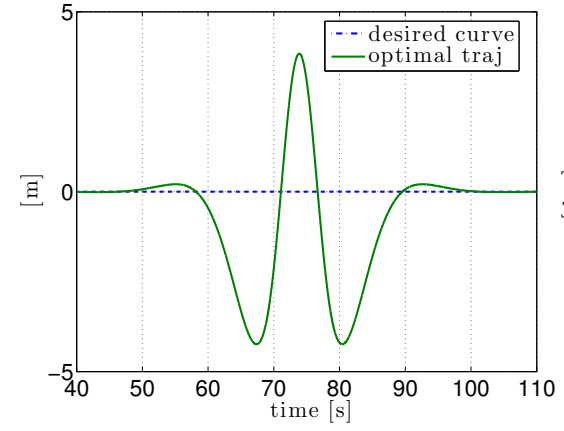

(a) $e_{y}$.

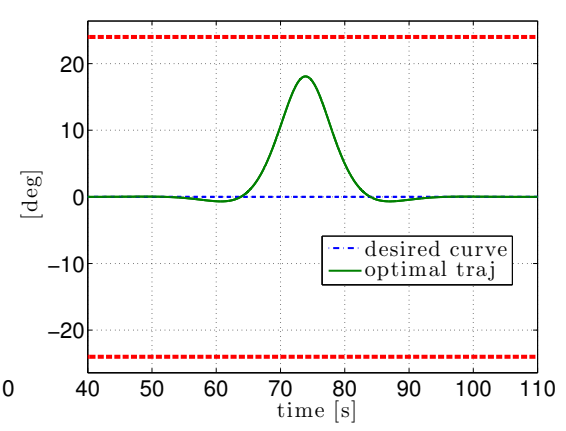

(b) $\phi_{A}$.

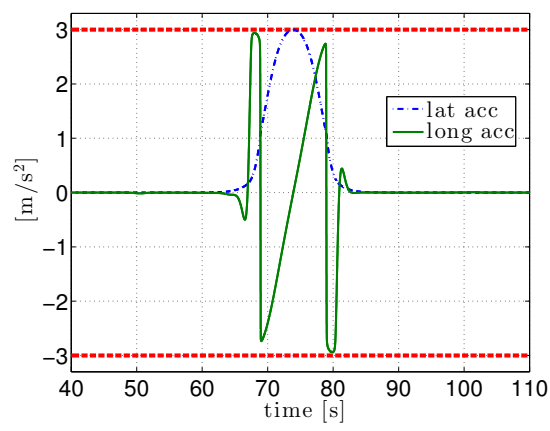

(c) $u_{4}$ vs $a_{\text {lat }}$.

Fig. 13. Rendezvous with coupled longitudinal and lateral motion for $k_{a g g r}=1$ (a) lateral error coordinate, (b) UAV roll angle, and (c) UGV longitudinallateral acceleration. Constraints are in dashed line.

factor becomes $n_{l f}=\frac{\cos \gamma_{A}}{\cos \phi_{A}}$. It is evident that the roll angle is constrained by $\arccos \frac{\cos \gamma_{A}}{n_{\text {lf max }}}$ which turns out to be less than $\phi_{\max }$. This explains the no-zero lateral error coordinate.

This computation allows us to highlight the coupled UAVUGV dynamics. Indeed, for $k_{\text {aggr }}=1$, the UAV roll reaches $18 \mathrm{deg}$ (as discussed before, its maximum value) and the UGV lateral acceleration is at the maximum value, $a_{\max }$, at exactly the same time $t=73.8 \mathrm{sec}$, see Figure $13 \mathrm{~b}$ and $13 \mathrm{c}$, respectively.

Finally, although the desired curve is based on the decoupled UAV-UGV dynamics, we are able to predict the rendezvous time. The sequence of rendezvous time is $111.8 \mathrm{sec}, 86.9 \mathrm{sec}$, $71.1 \mathrm{sec}, 60.3 \mathrm{sec}, 52.4 \mathrm{sec}$ for $k_{\text {aggr }}=0,0.25,0.5,0.75,1$, respectively, as predicted by (16).

\section{Simulations with high fidelity UAV model}

The proposed optimization-based strategy allows us to efficiently solve the rendezvous problem with a low computational effort by using a suitable vehicle model (introduced in Sections II-A), which still captures the main features of a real UAV. Next we validate our results by providing the optimal trajectory obtained from the PRONTO solver to a complex UAV model under time varying wind disturbances.

In particular, we use a complete 6-DOF dynamic model based on a Zagi flying wing airplane, [24]. This model is based on a fairly complex UAV model: six-degree-of-freedom, twelve-state equations of motion with quasi-linear aerodynamic and propulsion models, including low-level controllers (i.e., autopilot). Given the computed (local) optimal trajectory 


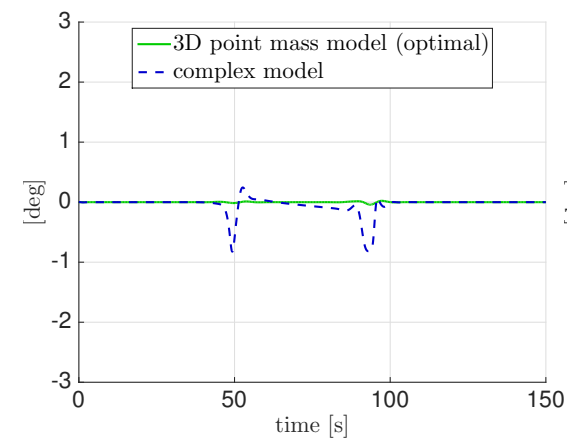

(a) $\phi$.

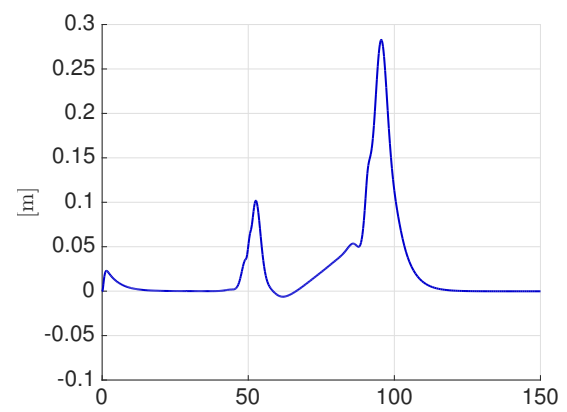

(d) Altitude error

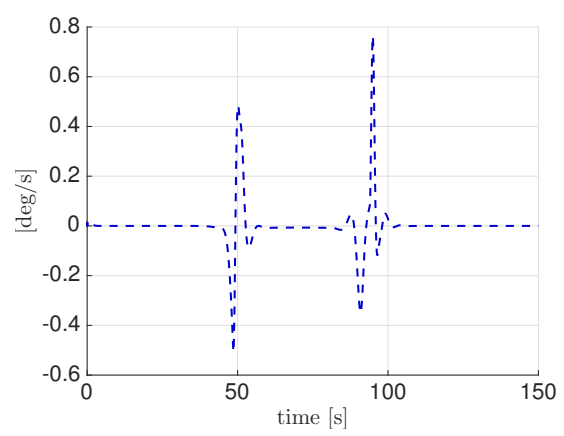

(g) Roll rate.

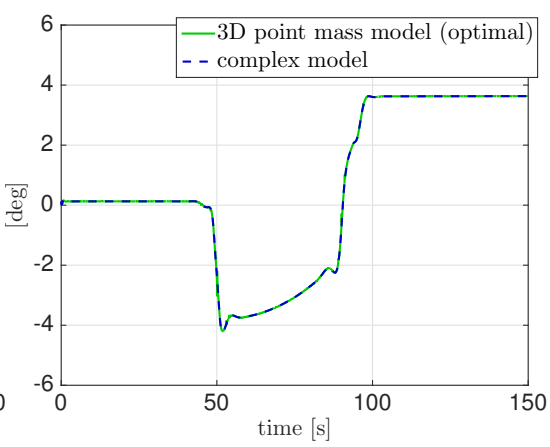

(b) $\theta$.

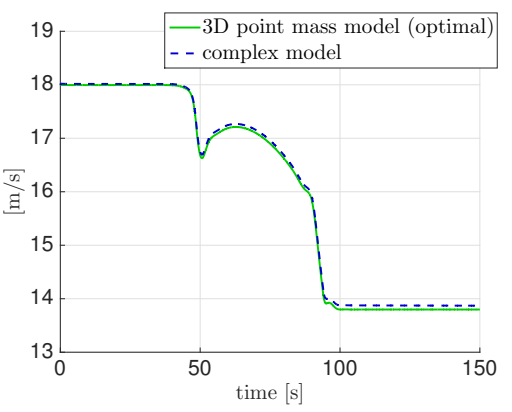

(e) $v_{g}$.

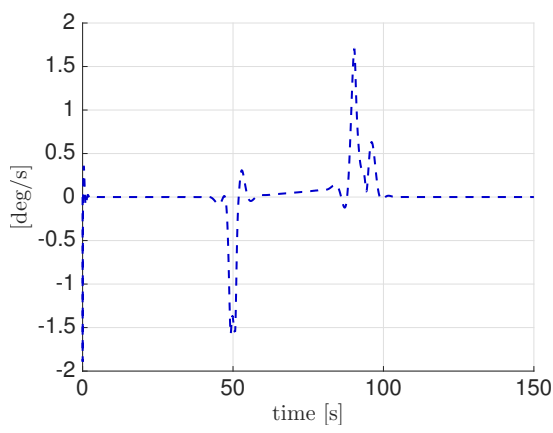

(h) Pitch rate.

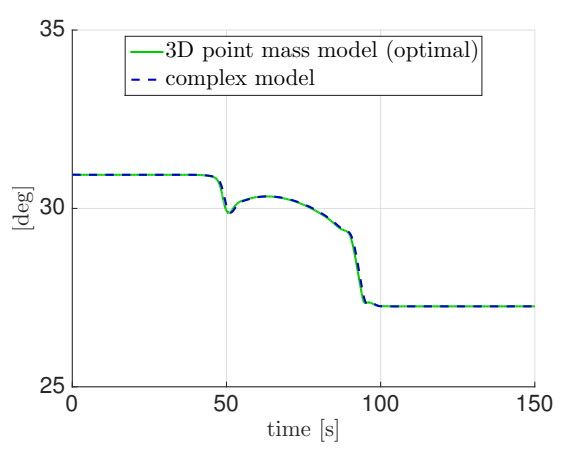

(c) $\psi$.

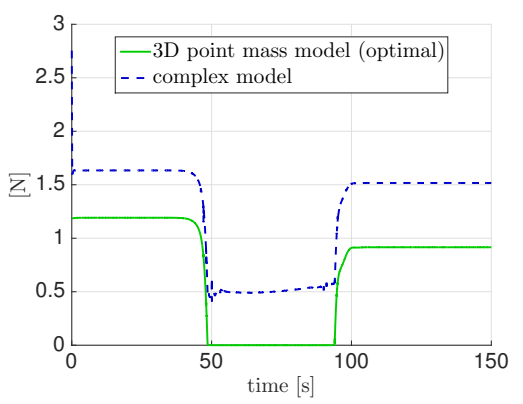

(f) $u_{1}$.

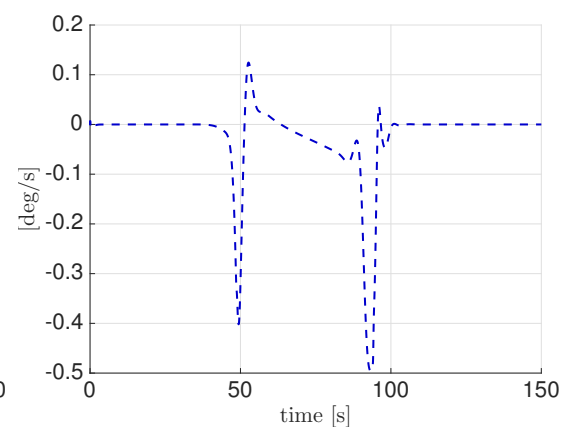

(i) Yaw rate.

Fig. 14. Simulation on a 6-DOF, 12-state equations of motion, Zagi flying wing aircraft (complex model) by using the optimal values obtained by the PRONTO solver as reference signals. Comparison between the 3D point mass model (green line) and the complex model (dashed blue line): roll (a), pitch (b) and yaw (c) angles, height (d), ground speed (e), thrust (f). In (g), (h), and (i) the roll rate, pitch rate and yaw rate, respectively, of the complex model.

for the straight line scenario, $k_{\text {aggr }}=1$ (shown in Figure 8, blue lines), the flight path angle, course angle, air-speed, and altitude are set as reference signals to the autopilot of the complex UAV model. The response of the UAV for the complete flight mission is shown in Figure 14. We can see that the UAV tracks the reference values very well. For example, we observe that the altitude error is less than $0.3 \mathrm{~m}$, see Figure 14d. However, some differences between the 3D point mass model and the complex model trajectories can be observed. This is due to the fact that the complex model has controller delays and saturations, complex aerodynamics and propulsion features playing during the flight. This is confirmed by the thrust profile shown in Figure 14f. For the complex vehicle model, we show the force due to thrust that is $F_{\text {thrust }}=\frac{1}{2} \rho S_{\text {prop }} C_{\text {prop }}\left(\left(k_{\text {motor }} \delta_{t}\right)^{2}-v_{a}^{2}\right)$, [24], where $\delta_{t}$ is the control input for the throttle deflection. In order to track the desired airspeed, the thrust has to compensate the drag force which takes into account additional terms due to pitch rate and elevator (which are not part of the 3D point mass model). Finally, we highlight that, during the landing phase, the UAV is aligned along the UGV path and hence the main components that are involved during this landing phase are the altitude, pitch and the ground speed. From the Figures $14 \mathrm{~g}, 14 \mathrm{~h}, 14 \mathrm{i}$, we can see that roll and yaw angular rates are almost zero and hence their contribution during the landing phase is negligible. The only contribution comes from the pitch rate which has effect on the flight path angle. The results confirm that the computed rendezvous trajectories of the 3-D point mass model are "close" to the trajectories of the complex 6-DOF dynamic model based on a Zagi flying wing airplane. This comparison gives a strong evidence of the efficiency of our strategy in dealing with the rendezvous problem. 


\section{COnClusions}

In this paper we proposed an optimal control approach for the refueling problem of fixed-wing UAVs using a UGV as a refueling unit. We provided a rigourous optimal control problem formulation for UAV rendezvous with the moving UGV and we addressed the optimal control problem by using a trajectory-tracking approach. Based on a nonlinear optimal control solver, we proposed an optimal control based strategy which allows us to compute optimal feasible trajectories for both UAV and UGV. By changing the aggressiveness index in our proposed strategy, we are able to compute aggressive trajectories (i.e., several constraints are active while the UAV is approaching the UGV) or very smooth ones. A key property of the proposed approach is that we are able to predict and, therefore, select (in form of tuning knob) the time to rendezvous, which is an important performance feature of the UAV trajectory. We provided numerical computations showing the effectiveness of the proposed approach. We have validated the results by using a fairly complex UAV model, [24]. The combination of the proposed rendezvous problem with the proposed strategy has been found to perform well in the presence of unmodeled dynamics. Future directions of research will include field tests where the obtained optimal trajectories are feed as reference trajectories to the trajectory tracking algorithms that are running on the vehicles to perform the rendezvous task. Another direction could be to include the UGV path into the problem formulation for jointly designing the trajectories for UAV and UGV which could be useful for applications like UAV landing on the UGV taking terrain restrictions into account. Further extension can also include UGV motion on a terrain.

\section{APPENDIX \\ MODEL PARAMETERS}

The UAV parameters are based on the "Zagi" flying wing [24]: $m=1.56 \mathrm{~kg}, S=0.2589 \mathrm{~m}^{2}, b=$ $1.4224 \mathrm{~m}, C_{D 0}=0.01631, k_{D / L}=0.04525, \rho=$ $1.225 \mathrm{~kg} / \mathrm{m}^{3}$. The minimum and maximum airspeed, normal load, maximum thrust, roll angle, and coefficient lift are set as follows $v_{\min }=12 \mathrm{~m} / \mathrm{s}, v_{\max }=$ $20 \mathrm{~m} / \mathrm{s}, n_{\text {lf } \min }=0.95, n_{\text {lf } \max }=1.05, \gamma_{\min }=$ $-6 \mathrm{deg}, \gamma_{\max }=10 \mathrm{deg}, \phi_{\max }=24 \mathrm{deg}, u_{1 \max }=$ $2 \mathrm{~N}, u_{2 \max }=5 \mathrm{deg} / \mathrm{s}, u_{3 \max }=0.7$. The maximum acceleration of the UGV is $a_{\max }=3 \mathrm{~m} / \mathrm{s}^{2}$ and the maximum course angle is defined by $\bar{e}_{x}=\bar{e}_{y}=\bar{e}_{z}=30 \mathrm{~m}, \bar{e}_{\chi}=2 \mathrm{deg}$. The other aerodynamic coefficient are given in [24].

\section{REFERENCES}

[1] M. L. Fravolini, A. Ficola, G. Campa, M. R. Napolitano, and B. Seanor, "Modeling and control issues for autonomous aerial refueling for UAVs using a probe-drogue refueling system," Aerospace science and technology, vol. 8, no. 7, pp. 611-618, 2004.

[2] J. Valasek, K. Gunnam, J. Kimmett, J. L. Junkins, D. Hughes, and M. D. Tandale, "Vision-based sensor and navigation system for autonomous air refueling," Journal of Guidance, Control, and Dynamics, vol. 28, no. 5, pp. 979-989, 2005.

[3] G. Campa, M. R. Napolitano, and M. L. Fravolini, "Simulation environment for machine vision based aerial refueling for UAVs," IEEE Transactions on Aerospace and Electronic Systems, vol. 45, no. 1, pp. 138-151, Jan 2009.
TABLE I

NOMENCLATURE

$p_{i}=\left[x_{i}, y_{i}, z_{i}\right]^{T}$
$v_{i}$
$\chi_{i}$
$\gamma_{A}, \phi_{A}, \psi_{A}$
$v_{a}$
$\gamma_{a}$
$T$
$D$
$L$
$m$
$g$
$\rho$
$S$
$C_{L}$
$C_{D}$
$C_{D_{0}}$
$k_{D} / L$
$n_{l f}$
$\alpha$
$a_{l o n}, a_{l a t}$
$\sigma_{G}$
$s_{G}$
$e=\left[e_{x}, e_{y}, e_{z}\right]^{T}$
$e_{\chi}, e_{\gamma}, e_{\phi}$
$e_{v}$
$v_{w}$
$w_{x}, w_{y}, w_{z}$

UAV $(i=A)$ and $\operatorname{UGV~}(i=G)$ position, $m$

UAV $(i=A)$ and UGV $(i=G)$ ground-speed, $m / s$ UAV $(i=A)$ and UGV $(i=G)$ course angle, rad

UAV flight path angle, roll angle, heading angle, $\mathrm{rad}$ UAV airspeed, $\mathrm{m} / \mathrm{s}$

UAV air-flight path angle, $\mathrm{rad}$

Thrust, $N$

Drag force, $N$

Lift force, $N$

Mass, $k g$

Gravitational acceleration, $\mathrm{m} / \mathrm{s}^{2}$

Air density, $\mathrm{kg} / \mathrm{m}^{3}$

Surface area of the wing, $m^{2}$

Lift coefficient

Drag coefficient

Drag coefficient at zero lift

Induced drag factor

Load factor

Angle of attack, $\mathrm{rad}$

UGV longitudinal and lateral acceleration, $\mathrm{m} / \mathrm{s}^{2}$

UGV path curvature, $1 / m$

UGV path coordinate, $m$

Longitudinal, lateral, and vertical error coordinates, $m$

Course angle, flight path, and roll error angles, $\mathrm{rad}$

Speed error, $\mathrm{m} / \mathrm{s}$

Wind speed, $\mathrm{m} / \mathrm{s}$

$w_{x}, w_{y}, w_{z} \quad$ Wind velocity components in the inertial frame, $\mathrm{m} / \mathrm{s}$

[4] J. W. Nichols, L. Sun, R. W. Beard, and T. McLain, "Aerial rendezvous of small unmanned aircraft using a passive towed cable system," Journal of Guidance, Control, and Dynamics, vol. 37, no. 4, pp. 1131-1142, 2014.

[5] L. Sun, J. D. Hedengren, and R. W. Beard, "Optimal trajectory generation using model predictive control for aerially towed cable systems," Journal of Guidance, Control, and Dynamics, vol. 37, no. 2, pp. 525539,2014

[6] L. Cantelli, M. Mangiameli, C. Melita, and G. Muscato, "UAV/UGV cooperation for surveying operations in humanitarian demining," IEEE International Symposium on Safety, Security, and Rescue Robotics (SSRR), pp. 1-6, 2013.

[7] B. Grocholsky, J. Keller, V. Kumar, and G. Pappas, "Cooperative air and ground surveillance," IEEE Robot Automation Magazine, vol. 13, no. 3, pp. 16-25, 2006.

[8] J. Sauter, R. Matthews, J. Robinson, J. Moody, and S. Riddle, "Swarming unmanned air and ground systems for surveillance and base protection,” AIAA Infotech@Aerospace Conference, pp. AIAA-2009-1850, Apr 2009.

[9] H. Yu, R. W. Beard, M. Argyle, and C. Chamberlain, "Probabilistic path planning for cooperative target tracking using aerial and ground vehicles," in American Control Conference (ACC), 2011, pp. 4673-4678.

[10] P. Tokekar, J. Vander Hook, D. Mulla, and V. Isler, "Sensor planning for a symbiotic UAV and ugv system for precision agriculture," in IEEE/RSJ International Conference on Intelligent Robots and Systems (IROS), 2013, pp. 5321-5326.

[11] J. Kampoon and A. Dogan, "Guidance of receiver aircraft to rendezvous with tanker in the presence of wind," AIAA Guidance, Navigation, and Control Conference, pp. AIAA-2010-8326, Aug 2010.

[12] B. Burns, P. Blue, and M. Zollars, "Autonomous control for automated aerial refueling with minimum-time rendezvous," AIAA Guidance, Navigation and Control Conference and Exhibit, pp. AIAA-2007-6739, Aug 2007.

[13] T. McLain and R. Beard, "Trajectory planning for coordinated rendezvous of unmanned air vehicles," AIAA Guidance, Navigation, and Control Conference and Exhibit, pp. AIAA-2000-4369, Aug 2000.

[14] N. Harl, "Coordinated rendezvous of unmanned air vehicles: A sliding mode approach," AIAA Guidance, Navigation and Control Conference and Exhibit, pp. AIAA-2008-6318, Aug 2008.

[15] T. Yamasaki and S. Balakrishnan, "Sliding mode based pure pursuit guidance for UAV rendezvous and chase with a cooperative aircraft," in American Control Conference (ACC), 2010, pp. 5544-5549.

[16] T. W. Carnes, T. M. Bakker, and R. H. Klenke, "A fully parameterizable implementation of autonomous take-off and landing for a fixed wing 
UAV," AIAA Guidance, Navigation, and Control Conference, pp. AIAA2015-0603, Jan 2015.

[17] H. Kim, M. Kim, H. Lim, C. Park, S. Yoon, D. Lee, H. Choi, G. Oh, J. Park, and Y. Kim, "Fully autonomous vision-based net-recovery landing system for a fixed-wing UAV," IEEE/ASME Transactions on Mechatronics, vol. 18, no. 4, pp. 1320-1333, 2013.

[18] J. Daly, Y. Ma, and S. Waslander, "Coordinated landing of a quadrotor on a skid-steered ground vehicle in the presence of time delays," in IEEE/RSJ International Conference on Intelligent Robots and Systems (IROS), 2011, pp. 4961-4966.

[19] B. Barber, T. McLain, and B. Edwards, "Vision-based landing of fixed-wing miniature air vehicles," Journal of Aerospace computing, Information, and Communication, vol. 6, no. 3, pp. 207-226, 2009.

[20] A. L. Smith, "Proportional navigation with adaptive terminal guidance for aircraft rendezvous," Journal of Guidance, Control, and Dynamics, vol. 31, no. 6, pp. 1832-1836, Nov 2008.

[21] A. Ratnoo, "Variable deviated pursuit for rendezvous guidance," Journal of Guidance, Control, and Dynamics, vol. 38, no. 4, pp. 787-792, Apr 2015.

[22] J. I. Lee, I. S. Jeon, and M. J. Tahk, "Guidance law to control impact time and angle," IEEE Transactions on Aerospace and Electronic Systems, vol. 43, no. 1, pp. 301-310, January 2007.

[23] C. H. Lee, M. J. Tahk, and J. I. Lee, "Generalized formulation of weighted optimal guidance laws with impact angle constraint," IEEE Transactions on Aerospace and Electronic Systems, vol. 49, no. 2, pp. 1317-1322, APRIL 2013.

[24] R. W. Beard and T. W. McLain, Small unmanned aircraft: Theory and practice. Princeton University Press, 2012, Chapter 2, Chapter 9.

[25] A. Rucco, P. Sujit, A. P. Aguiar, and J. Sousa, "Optimal UAV rendezvous on a UGV," AIAA Guidance, Navigation, and Control Conference, pp. AIAA-2016-0895, San Diego, CA, 2016.

[26] F. Bayer and J. Hauser, "Trajectory optimization for vehicles in a constrained environment," in IEEE Conference on Decision and Control, 2012, pp. 5625-5630.

[27] E. Velenis and P. Tsiotras, "Minimum-time travel for a vehicle with acceleration limits: Theoretical analysis and receding-horizon implementation," Journal of Optimization Theory and Applications, vol. 138, no. 2, pp. 275-296, 2008.

[28] A. Rucco, G. Notarstefano, and J. Hauser, "An efficient minimumtime trajectory generation strategy for two-track car vehicles," IEEE Transactions on Control Systems Technology, vol. 23, no. 4, pp. 15051519, July 2015.

[29] J. Hauser, "A projection operator approach to the optimization of trajectory functionals," in IFAC world congress, vol. 15, 2002, p. 310.

[30] J. Hauser and A. Saccon, "A barrier function method for the optimization of trajectory functionals with constraints," in IEEE Conference on Decision and Control, 2006, pp. 864-869.

[31] A. Saccon, J. Hauser, and A. P. Aguiar, "Optimal control on lie groups: The projection operator approach," IEEE Transactions on Automatic Control, vol. 58, no. 9, pp. 2230-2245, 2013.

[32] J. W. Langelaan, N. Alley, and J. Neidhoefer, "Wind field estimation for small unmanned aerial vehicles," Journal of Guidance, Control, and Dynamics, vol. 34, no. 4, pp. 1016-1030, 2011.

[33] Z. Jiang and R. Ordóñez, "On-line robust trajectory generation on approach and landing for reusable launch vehicles," Automatica, vol. 45, no. 7 , pp. 1668-1678, 2009.

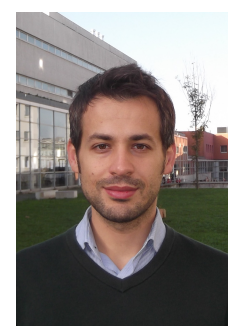

Alessandro Rucco received the Master's degree in automation engineering and the Ph.D. degree in information engineering from the Università del Salento, Lecce, Italy, in 2009 and 2014, respectively. He was Visiting Student at the University of Paris Sud Supélec (March-April 2010) and at the University of Colorado Boulder (January-September 2012). He was Team Leader of the VI-RTUS team winning the International Student Competition Virtual Formula 2012. From August 2014 to September 2016, he was a Post-Doctoral Researcher at the Department of Electrical and Computer Engineering, University of Porto, Portugal. Currently, he is a Post-Doctoral Researcher at the Department of Engineering, Università del Salento. His research interests include distributed optimization, nonlinear optimal control, modeling, trajectory optimization, and high-performance maneuvering for car and aerial vehicles.

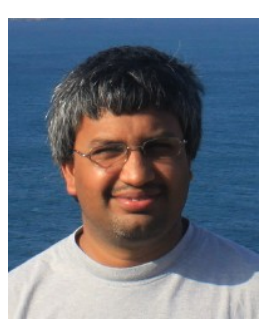

P.B. Sujit is Lecturer at Loughborough University, UK and Assistant Professor at the IIIT Delhi, New Delhi, India. He received the bachelor's degree from Bangalore University, India in 1998, Master's degree from Visveswaraya Technological University, India in 2002, and Ph.D. from the Indian Institute of Science, Bangalore, India in 2006. He is an Editor for Journal of Intelligent \& Robotic Systems. His research interests include unmanned aerial and underwater vehicles, multi-robot systems, and humanrobot interaction.

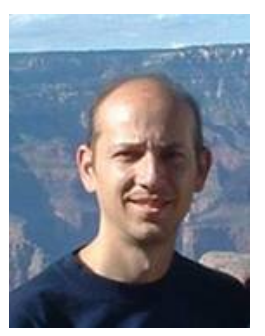

A. Pedro Aguiar received the Licenciatura, M.S. and $\mathrm{Ph} . \mathrm{D}$. in Electrical and Computer Engineering from the Instituto Superior Técnico (IST), Technical University of Lisbon, Portugal in 1994, 1998 and 2002, respectively. Currently, Dr. Aguiar holds an Associate Professor position with the Department of Electrical and Computer Engineering (DEEC), Faculty of Engineering, University of Porto (FEUP). From 2002 to 2005 , he was a post-doctoral researcher at the Center for Control, DynamicalSystems, and Computation at the University of California, Santa Barbara (UCSB). From 2005 to 2012, he was a senior researcher with the Institute for Systems and Robotics at IST, and an invited assistant professor with the Department of Electrical and Computer Engineering, IST. His research interests include modeling, control, navigation, and guidance of autonomous robotic vehicles, nonlinear control, switched and hybrid systems, tracking, path-following, performance limitations, nonlinear observers, the integration of machine vision with feedback control, networked control, and coordinated/cooperative control of multiple autonomous robotic vehicles.

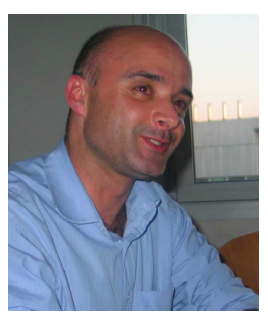

João Borges de Sousa is an Assistant Professor with the DEEC at FEUP and Director of the LSTS Laboratory. His research interests include unmanned vehicles, control architectures, control, and coordination. He has been leading the design, implementation, and deployment of advanced unmanned air and ocean vehicle systems in projects funded by the FCT, the Portuguese MoD, the Portuguese Innovation Agency (ADI), the European Union (FP6/FP7/H2022), NATO and, ONR and DARPA from USA. In 2006 the laboratory received the national BES Innovation National Award for the design of the Light Autonomous Underwater Vehicle. In 2007 he received an outstanding teaching award from Porto University. He has authored approximately 340 scientific papers.

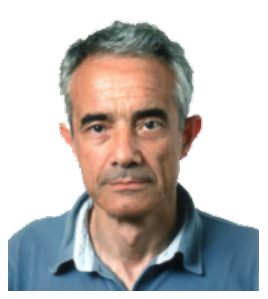

F. Lobo Pereira is Full Professor at DEEC in FEUP, Scientific Coordinator of the SYSTEC Research Unit, and Director of the Institute for System and Robotics Porto. He received his Ph.D. degree in Control Theory from Imperial College of Science and Technology from London University in 1986, and the Habilitation Title in Automation and Control from Porto University in 1997. His research work falls in the following areas: Control Theory (Dynamic Optimization, Impulsive Control), Hybrid Systems, and Coordinated Control, and their application in the control of autonomous and/or networked systems. He has been involved in a large number of European and national research projects and has been EU expert for the evaluation and execution follow-up of R\&D project proposals. He is in the editorial board of the Journal Computational and Applied Mathematics and Trends in Applied Mathematics. He has co-authored more than 300 scientific articles in peer-reviewed international journals and conferences. 